\title{
Perfil de Prescrição de Estatinas e de Níveis Lipêmicos em Ambulatórios de Hospital Terciário Público
}

\author{
Statins Prescriptions and Lipid Levels in a Tertiary Public Hospital
}

André Schmidt, ${ }^{\circledR}$ Henrique Turin Moreira, ${ }^{1}$ Gustavo Jardim Volpe, ${ }^{1}$ Vamberto B. Foschini, ${ }^{1}$ Thiago Florentino Lascala, ${ }^{1}$ Minna Moreira Dias Romano, ${ }^{1}$ Marcus Vinícius Simões, ${ }^{1}$ José Ernesto dos Santos, ${ }^{1}$ Benedito Carlos Maciel, ${ }^{1}$ José Antonio Marin-Neto ${ }^{1}$

Universidade de São Paulo Faculdade de Medicina de Ribeirão Preto (HCFMRP-USP), ${ }^{1}$ Ribeirão Preto, SP - Brasil

\section{Resumo}

Fundamento: $\mathrm{O}$ surgimento de nova classe de medicamentos com elevada capacidade de reduzir o LDL-colesterol (LDL-c) renovou o interesse na caracterização da hipercolesterolemia familiar (HF). Pouco se conhece do perfil lipídico de pacientes em atendimento terciário em nosso meio para caracterizar a real ocorrência de HF, que começa a ser suspeitada com níveis de LDL-c acima de $190 \mathrm{mg} / \mathrm{dL}$.

Objetivos: O estudo avaliou o perfil lipídico (colesterol total [CT] e LDL-c) de pacientes de hospital público terciário.

Métodos: Estudo retrospectivo de avaliação de prescrições de estatinas e resultados dos lipídios. O nível de significância foi estabelecido em $5 \%$.

Resultados: Em 1 ano, 9.594 indivíduos receberam prescrição ambulatorial de estatinas, 51,5\% do gênero feminino, idade média de $63,7 \pm 12,9$ anos (18 a 100 anos). Trinta e duas especialidades prescreveram estatinas, sendo a cardiologia responsável por $43 \%$. Cerca de $15 \%$ das prescrições não tinham dosagem recente de CT, e $1.746(18,0 \%)$ não apresentavam resultado recente de LDL-c. A ocorrência de LDL-c $>130 \mathrm{mg} / \mathrm{dL}$ e $<190 \mathrm{mg} / \mathrm{dL}$ ocorreu em 1.643 $(17,1 \%)$ casos, e $228(2,4 \%)$ apresentaram LDL-c $\geq 190 \mathrm{mg} / \mathrm{dL}$ dentre os que utilizavam estatinas nas diversas doses. Apenas duas estatinas foram utilizadas: sinvastatina e atorvastatina, e a primeira foi prescrita em $77,6 \%$ das receitas.

Conclusão: Nesta coorte transversal de hospital terciário, foi possível verificar que a prescrição de estatinas é disseminada, mas que a obtenção de metas adequadas de CT e LDL-c não é atingida em grande percentual, e que há, possivelmente, significativo contingente de portadores de HF que necessitariam ser investigados por suas implicações prognósticas. (Arq Bras Cardiol. 2021; 116(4):736-741)

Palavras-chave: Inibidoresde Hidroimetilglutaril CO Redutases; Dislipidemias; Hiperlipoproteinemia Tipo Il; Hipercolesterolemia Familiar/terapia; Perfil Lipídico; Hospital Público; Colesterol.

\begin{abstract}
Background: The development of a new class of medications that are highly capable of reducing LDL-cholesterol renewed the interest in the characterization of familial hypercholesterolemia patients. Nevertheless, little is known about the lipid profile of patients in tertiary healthcare centers in Brazil in order to better estimate the real occurrence of familial hypercholesterolemia, with initial suspect of LDL-cholesterol levels above $190 \mathrm{mg} / \mathrm{d} / \mathrm{L}$.

Objectives: This study evaluated the lipid profile (total cholesterol and LDL-cholesterol) in ambulatory patients from a general tertiary public hospital.

Methods: Retrospective study comparing prescriptions of statins and lipid profile results. The significance level was established in 5\%.

Results: In one year, 9,594 individuals received statin prescriptions, of whom 51.5\% were females and the mean age was $63.7 \pm 12.9$ years-old (18 to 100 years-old). Thirty-two medical specialties prescribed statins. Cardiology was responsible for $43 \%$ of the total. Nearly $15 \%$ of those patients with a prescription did not have a recent total cholesterol result and 1,746 (18\%) did not have a recent LDL-cholesterol measurement. The occurrence of the latter between 130 and $190 \mathrm{mg} / \mathrm{dL}$ was present in 1,643 (17.1\%) individuals, and 228 (2.4\%) patients had an LDLcholesterol $\geq 190 \mathrm{mg} / \mathrm{dL}$ among those using statins at distinct doses. Only two statins were used: simvastatin and atorvastatin. The first was prescribed in $77.6 \%$ of the prescriptions.
\end{abstract}

Correspondência: André Schmidt•

Universidade de São Paulo Faculdade de Medicina de Ribeirão Preto - Centro de Cardiologia - HCFMRP-USP Av. Bandeirantes, 3900. CEP 14048-900, Ribeirão Preto, SP - Brasil

E-mail: aschmidt@fmrp.usp.br

Artigo recebido em 06/08/2019, revisado em 25/02/2020, aceito em 16/03/2020

DOI: https://doi.org/10.36660/abc.20190513 
Conclusion: In this cross-sectional cohort at a tertiary general hospital, statins have been widely prescribed but with little success in achieving recognized levels of control. There is probably a significant number of FH individuals in this cohort that need to be properly diagnosed in order to receive adequate treatment due to its prognostic implications. (Arq Bras Cardiol. 2021; 116(4):736-741)

Keywords: Hydroxymethylglutaryl CO-Reductase Inhibitors; Dyipidemias; Hyperpoprotein Type II. Hypercholesterolemia Familial/therapy; Cholesterol; Public Hospital; Lipid Profile.

Full texts in English - http://www.arquivosonline.com.br

\section{Introdução}

Apesar de metanálise recente sugerir ser de 1:250 a incidência de HF na população geral, ${ }^{1}$ desconhece-se a real prevalência específica de casos com alterações extremas da colesterolemia em serviços públicos ambulatoriais terciários de nosso país. Esses serviços concentram, em geral, os casos com mais comorbidades e maior gravidade clínica.

Alguns estudos avaliaram a custo-efetividade da utilização de estatinas pelo Sistema Único de Saude (SUS); ;,3 contudo, a aderência ao tratamento foi pouco estudada em grupos seletos (mulheres), atingindo 15,5\% em pequena série. ${ }^{4}$ Com a recente incorporação ao arsenal terapêutico de novos medicamentos altamente eficazes no controle de hipercolesterolemia, , $^{6}$ ainda que de elevado custo, os hospitais terciários tornaram-se ponto de convergência de pacientes com perfil lipídico muito alterado, visando à sua prescrição pelo sistema público. No entanto, pouco se conhece do perfil lipídico e do tratamento de pacientes em seguimento ambulatorial nessas instituições.

O objetivo deste estudo consiste em relatar o estado atual da prescrição de estatinas em um hospital público de nível terciário e o grau de controle da dislipidemia assim obtido, além de verificar a possível existência de casos sugestivos de HF (LDL-C > 190mg/dL) mesmo em uso de estatinas.

\section{Material e métodos}

Trata-se de estudo de coorte transversal. Uma coleta sistematizada foi obtida no prontuário eletrônico da Instituição cotejando todos os indivíduos com idade igual ou superior a 18 anos que receberam prescrição ambulatorial de uma estatina no Hospital das Clínicas da Faculdade de Medicina de Ribeirão Preto da Universidade de São Paulo (HCFMRP-USP), hospital terciário de ensino, durante o ano de 2016. Além disso, foram coletadas as dosagens de CT e/ou LDL-c no ano de 2016, posteriormente realizadas ambulatorialmente. Para aqueles que realizaram mais que um exame de lipídios naquele ano, foi considerado o último exame. Idade e gênero foram coletados. Foram também registrados o medicamento prescrito e a dose utilizada, bem como a clínica que a prescreveu quando da realização do último exame. Considerando ser uma busca eletrônica direcionada, não foram coletados dados relativos a comorbidades ou clínicos/antropométricos. Esse estudo foi aprovado pelo Comitê de Ética em Pesquisa local e registrado com o número CAAE 16516819.0.0000.5440.

\section{Análise estatística}

Foi realizada análise descritiva de tais dados, que foram expressos em média e desvio padrão quando a distribuição foi considerada normal pelo teste de Kolmogorov-Smirnov. Variáveis qualitativas foram expressas em porcentagem. Teste de correlação de Pearson foi utilizado para correlacionar os valores de CT e LDL-c. Teste t de Student não pareado foi utilizado para comparação de idades utilizada.

Foi utilizado o programa SPSS v.25 (IBM Corporation, EUA), e o nível de significância foi estabelecido em $<5 \%$.

\section{Resultados}

\section{Prescrições realizadas}

Ao longo de 2016, 9.594 indivíduos receberam prescrição ambulatorial de estatinas na Instituição. Houve discreto predomínio do gênero feminino com 4.942 (51,5\%) prescrições, e a idade média foi de $63,7 \pm 12,9$ anos (18 a 100 anos). Nesses pacientes, foram identificados 8.110 (84,8\%) resultados de CT e $7.848(82,0 \%)$ de LDL-c, indicando que $1.484(15,2 \%)$ indivíduos receberam prescrição sem dosagem recente de CT e $1.746(18,0 \%)$ pacientes não apresentavam resultado recente de LDL-c. Ressalta-se que, em todas as especialidades, houve casos de prescrições sem dosagens; no entanto, na cirurgia vascular, isso foi mais saliente, com cerca de $75 \%$ das receitas emitidas sem dosagem de LDL-c.

No que tange as 32 especialidades médicas que as prescreveram, a cardiologia foi responsável por $43,5 \%$ das prescrições, seguida pela cirurgia vascular com $9,2 \%$ e nefrologia com $8,6 \%$. O restante (32\%) ficou distribuído em todas as outras especialidades presentes em um hospital terciário público, sendo a nutrologia responsável por apenas $106(1,1 \%)$ receitas ambulatoriais (Tabela 1$)$.

Tabela 1 - Distribuição das prescrições de estatinas por especialidade médica no ano de 2016

\begin{tabular}{lc}
\hline Especialidade & N (\%) \\
\hline Cardiologia & $4.160(43,5)$ \\
\hline Cirurgia vascular & $1.576(9,2 \%)$ \\
\hline Nefrologia & $819(8,6)$ \\
\hline Neurologia & $731(7,6)$ \\
\hline Geriatria & $657(6,9)$ \\
\hline Endocrinologia & $653(6,8)$ \\
\hline Nutrologia & $94(1,0)$ \\
\hline Outras 25 especialidades & $1.576(16,5)$ \\
\hline Total & $9.567(100)$ \\
\hline
\end{tabular}




\section{Perfil de lipídios}

Colesterol total médio da amostra foi de $174,4 \pm 49,5 \mathrm{mg} /$ $\mathrm{dL}$ variando entre 40,0 e $739,0 \mathrm{mg} / \mathrm{dL}$, enquanto o LDL-C médio foi de $101,1 \pm 40,0 \mathrm{mg} / \mathrm{dL}$, variando entre 4,0 e 635,0 $\mathrm{mg} / \mathrm{dL}$. Uma forte correlação entre os valores de CT e LDL-C foi observada $(r=0,94 ; p<0,001)$. A distribuição amostral dos valores obtidos para CT e LDL-c está resumida na Figura 1.

Verificou-se que as mulheres apresentaram níveis lipídicos significativamente mais alterados que os homens, tanto para CT $(183,3 \pm 49,9$ vs. $164,5 \pm 47,0 \mathrm{mg} / \mathrm{dL} ; \mathrm{p}<0,001)$ quanto para LDL-c (107,1 \pm 40,9 vs. 94,3 \pm 7,9mg/dL; $p<0,001)$, ainda que a idade média dos dois gêneros fosse semelhante $(63,65 \pm 13,56$ vs. $63,36 \pm 12,60 ; p=0,29)$.

A ocorrência de LDL-c > 130mg/dL e < 190mg/dL ocorreu em $1.643(17,1 \%)$ indivíduos com prescrição de estatina. Some-se a isso o fato de que $18,2 \%$ da amostra total não apresentava dosagem de LDL-c, apesar da prescrição de estatina ambulatorial. Portanto, possivelmente, um considerável percentual de indivíduos apresenta LDL-c acima do preconizado em diretrizes de prevenção primária, apesar do uso de estatinas e sem levar em consideração as entidades nosológicas concomitantes por eles apresentadas.

A

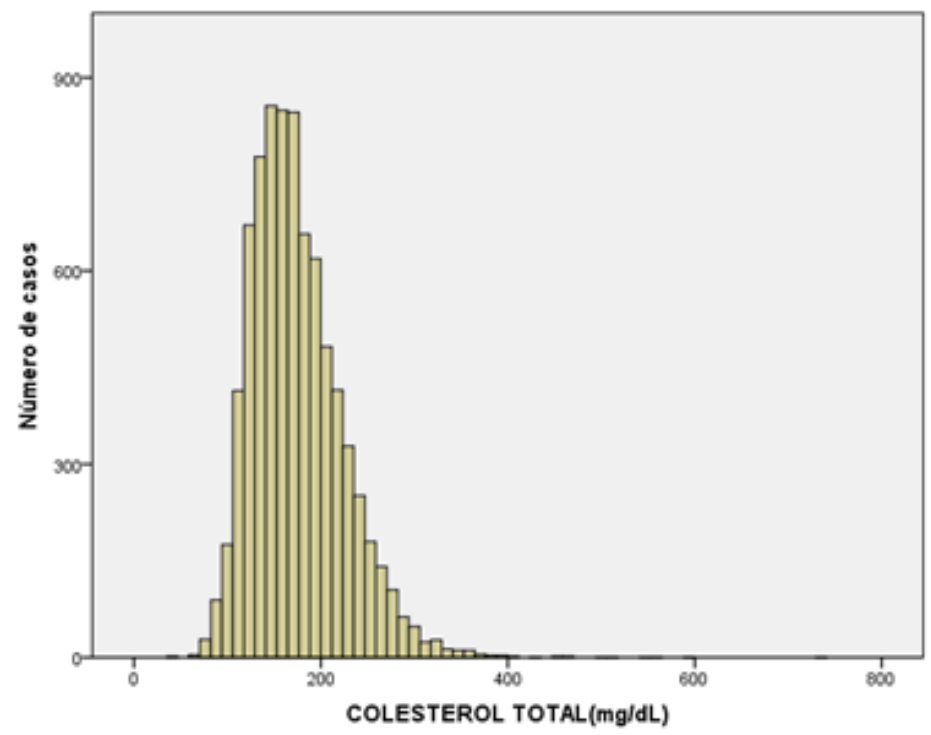

B

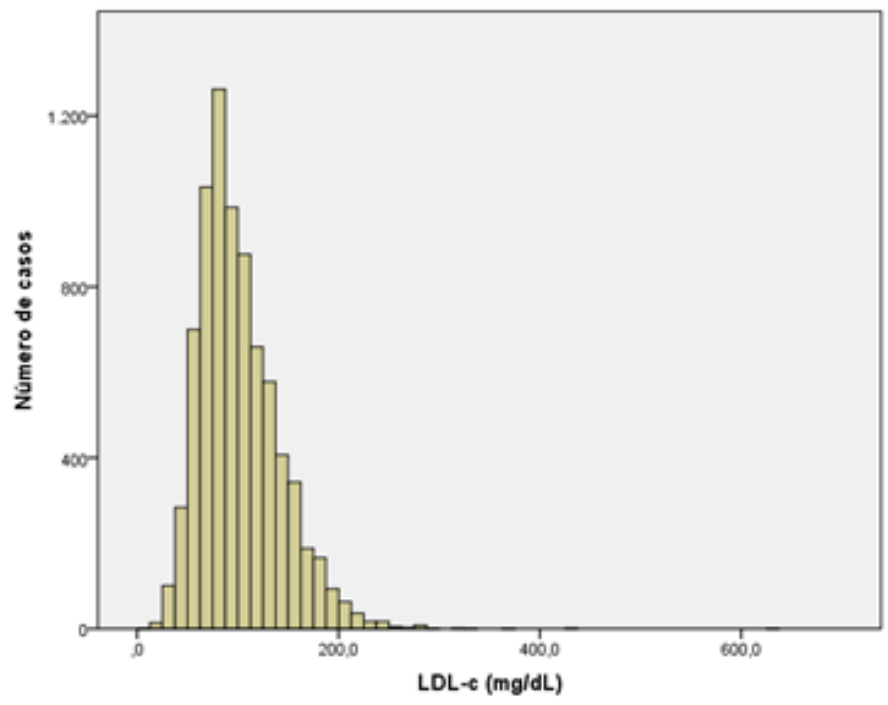

Figura 1 - Histogramas de distribuição de frequência dos valores absolutos de colesterol total (A) e LDL-c (B) na amostra de pacientes do Hospital das Clínicas da Faculdade de Medicina de Ribeirão Preto da Universidade de São Paulo (HCFMRP-USP) no ano de 2016. 
Finalmente, 228 (2,4\%) indivíduos apresentaram LDL-C $\geq 190 \mathrm{mg} / \mathrm{dL}$ dentre os que utilizavam estatinas nas diversas doses. Dois terços (152) eram do gênero feminino. Este grupo de pacientes apresenta idade média menor que a da amostra total ( $55 \pm 15$ anos vs. $63 \pm 13 p<0,05)$, podendo este ser um indicador a mais para sugerir a ocorrência de HF neste grupo de pacientes de um hospital terciário.

\section{Estatinas utilizadas}

Por se tratar de hospital público, apenas duas estatinas estão disponíveis para prescrição ambulatorial: sinvastatina e atorvastatina. A primeira foi prescrita em $7.474(77,6 \%)$ receitas. Sinvastatina na dose de $40 \mathrm{mg}$ foi prescrita em $3.760(39,3 \%)$ receitas, seguida por Sinvastatina 20mg em 3158 (33,0\%). Atorvastatina na dose de 40mg foi a terceira mais utilizada, em $1.087(11,4 \%)$ receitas ambulatoriais. A Tabela 2 apresenta os níveis de CT e LDL-c de acordo com o medicamento e dose utilizados.

Verificamos que, no caso da sinvastatina, a dose crescente, aparentemente, foi utilizada em casos mais resistentes, sugerindo que uma adequação da dose foi realizada. Para aqueles em uso de atorvastatina, verificamos que o nível médio do CT e LDL-c decresce de modo não estatisticamente significante $(p>0,05)$ com o incremento da dose até o nível de 40mg diários. A dose de $80 \mathrm{mg}$, utilizada em 3\% dos indivíduos, foi possivelmente utilizada em indivíduos com menor resposta ao seu uso, pois ambos os valores de CT e LDL-c foram significativamente maiores que os dos que receberam atorvastatina $40 \mathrm{mg}$ diários $(p<0,05)$. De modo geral, verifica-se que doses mais elevadas foram utilizadas, sugerindo a tentativa por um melhor controle da hipercolesterolemia.

Os níveis médios de CT e LDL-c foram menores significativamente $(p<0,05)$ nos pacientes recebendo prescrições pela especialidade de cardiologia comparativamente aos outros grupos ambulatoriais, sendo o único com CT médio abaixo de 170mg/dL e LDL-c médio abaixo de $95 \mathrm{mg} / \mathrm{dL}$ (Tabela 3).

\section{Discussão}

Neste estudo, pudemos verificar que a prescrição de estatinas em um hospital terciário do sistema público é bastante frequente, possivelmente pela densa concentração de pacientes com elevado número de comorbidades de ordem cardiovascular em seguimento na Instituição. Vannucchi et al., em nossa Instituição, nos anos 1970, descreveram o perfil lipídico em lipidogramas de cerca de 1.700 pacientes coletados durante período de cerca de 3 anos. Esses autores verificaram que $25,5 \%$ dos lipidogramas solicitados apresentavam alterações diagnósticas de pelo menos uma dislipidemia. Contudo, diante da inexistência de tratamentos específicos à época, nada foi discutido sobre o tópico da terapêutica. ${ }^{7} \mathrm{Na}$ literatura mundial, Pant et al. reportaram dados de perfil lipídico em hospital de nível terciário no Nepal. ${ }^{8}$ Esses autores utilizaram uma amostra de conveniência ambulatorial sem registro do uso de estatinas ou comorbidades e verificaram que, em 408 casos avaliados com idade média de 50 anos, os valores médios de LDL-c e CT eram 113 41 $\mathrm{mg} / \mathrm{dL}$ e $180 \pm 54 \mathrm{mg} / \mathrm{dL}$, respectivamente. Estudo na Turquia utilizou os resultados de lipidogramas para proceder à busca ativa de casos de HF, tendo verificado que muitos casos de elevação de LDL-c nem estavam recebendo tratamento. ${ }^{9}$

De modo original, nesta investigação, verificamos que a prescrição de estatinas ocorre de modo disseminado na Instituição. Tal fato indica o conhecimento do fator de risco cardiovascular, mas sem necessariamente visar a uma meta específica, pois há grande variabilidade nos valores médios em cada especialidade, notadamente naquelas lidando com doenças cardiovasculares (Divisão de Cirurgia Vascular do Departamento de Cirurgia e Departamento de Neurologia). Verificamos que a nutrologia, responsável por $1,1 \%$ das receitas emitidas, apresenta os níveis médios lipídicos mais elevados, fato possivelmente relacionado com a circunstância de que casos resistentes ou com HF são referenciados preferencialmente para esse ambulatório em nível terciário. Além disso, para essa amostra específica de pacientes, pode-se especular que, além de estatinas, outros medicamentos mais

Tabela 2 - Níveis séricos de colesterol total e sua fração LDL-c encontrados de acordo com o tipo e a dose da estatina. Pacientes com doses intermediárias não foram incluídos

\begin{tabular}{|c|c|c|c|}
\hline $\begin{array}{l}\text { Medicamento } \\
\text { e dose diária }\end{array}$ & Número de pacientes & $\begin{array}{c}\text { Colesterol total }(\mathrm{mg} / \mathrm{dL}) \\
\text { Média } \pm \mathrm{DP}\end{array}$ & $\begin{array}{l}\text { LDL-c }(\mathrm{mg} / \mathrm{dL}) \\
\text { Média } \pm \text { DP }\end{array}$ \\
\hline \multicolumn{4}{|l|}{ Sinvastatina } \\
\hline $10 \mathrm{mg}$ & 302 & $168 \pm 43$ & $96 \pm 36$ \\
\hline $20 \mathrm{mg}$ & 3.164 & $177 \pm 49$ & $103 \pm 40$ \\
\hline $40 \mathrm{mg}$ & 3.764 & $173 \pm 52$ & $100 \pm 41$ \\
\hline $80 \mathrm{mg}$ & 57 & $193 \pm 66$ & $110 \pm 34$ \\
\hline \multicolumn{4}{|l|}{ Atorvastatina } \\
\hline $10 \mathrm{mg}$ & 92 & $184 \pm 63$ & $109 \pm 53$ \\
\hline $20 \mathrm{mg}$ & 481 & $176 \pm 56$ & $100 \pm 42$ \\
\hline $40 \mathrm{mg}$ & 1.088 & $170 \pm 52$ & $97 \pm 41$ \\
\hline $80 \mathrm{mg}$ & 283 & $182 \pm 51$ & $109 \pm 43$ \\
\hline
\end{tabular}

DP: desvio padrão. 
Tabela 3 - Níveis séricos médios de colesterol total e sua fração LDL-c de acordo com a especialidade prescritora no ano de 2016

\begin{tabular}{lll}
\hline Especialidade & Colesterol total $\mathbf{( m g / d L})$ & LDL-c $(\mathbf{m g} / \mathbf{d L})$ \\
\hline Cardiologia & $166,1 \pm 45,1$ & $94,6 \pm 36,3$ \\
\hline Endocrinologia & $179,5 \pm 49,3$ & $103,8 \pm 40,3$ \\
\hline Geriatria & $171,6 \pm 45,0$ & $98,4 \pm 37,2$ \\
\hline Nefrologia & $183,3 \pm 58,5$ & $104,6 \pm 46,6$ \\
\hline Neurologia & $171,9 \pm 45,1$ & $104,5 \pm 36,9$ \\
\hline Vascular & $175,0 \pm 51,8$ & $104,2 \pm 39,4$ \\
\hline Nutrologia & $186,5 \pm 47,0$ & $112,7 \pm 41,1$ \\
\hline Outras & $192,5 \pm 53,4$ & $115,3 \pm 44,6$ \\
\hline
\end{tabular}

novos - como a ezetimiba, que reduz a absorção intestinal de colesterol, e os inibidores da proproteína convertase subtilisina/kexina tipo 9 (PCSK9) - ainda não estão disponíveis e devem ser necessários.

A utilização de atorvastatina foi modesta (22\%). Por ser necessária a prescrição mais elaborada e demorada, com inclusão dos resultados laboratoriais, pode ter havido subutilização. O encontro de elevado percentual de pacientes em uso de estatinas sem que haja pelo menos um exame anual de controle sugere que não são utilizados protocolos clínicos locais ou diretrizes publicadas. ${ }^{10}$ Podemos ainda constatar que, em grande número de pacientes, nenhum ajuste medicamentoso é realizado, e a dose utilizada é prescrita de modo "automático", ou seja, em critérios avalizados. Possivelmente, as doses em uso necessitariam ser ajustadas de modo periódico e coordenado, dentro de protocolos clínicos institucionais bem estabelecidos, visto que pacientes oriundos de ambulatórios vinculados fortemente com a ocorrência de doenças cardiovasculares, mas sem protocolos clínicos definidos, apresentam níveis médios significativamente maiores que aqueles observados nos ambulatórios de cardiologia, nos quais eles existem, sugerindo que o controle de fatores de risco é visto com enfoques distintos, ainda que inseridos em um mesmo contexto nosológico.

Apesar de os níveis médios globais estarem dentro de níveis aceitáveis para uma amostra da população geral, cabe lembrar que há um grande número de indivíduos com elevado risco cardiovascular nessa amostra, o que sugere a existência de espaço para maior redução dos valores médios. É possível ainda constatar uma elevada proporção de pacientes com valores muito elevados apesar da utilização de estatinas. Isso pode indicar um problema relativo à aderência ao tratamento. Como estão disponíveis gratuitamente na rede pública, não há por que discutir restrições financeiras, e, no período avaliado, não houve falta de medicamentos na rede assistencial.

Outro aspecto a ser ressaltado está no fato de que pacientes do gênero feminino apresentavam níveis lipídicos significativamente mais elevados que os do gênero masculino. A proporção de mulheres acima de 60 anos de idade com elevação do colesterol total é maior em várias séries, ${ }^{11,12}$ sem que uma clara explicação fosse oferecida. O papel da menopausa nesse incremento não pode ser desconsiderado. Embora especulativamente no contexto, é plausível supor que haja certa displicência com o ajuste medicamentoso em pacientes desse gênero no que tange ao controle de fatores de risco para doença arterial coronariana, algo também previamente relatado. ${ }^{13}$

O predomínio da utilização de sinvastatina possivelmente decorre da sua disponibilização pelo sistema único de saúde (SUS) em nível ambulatorial de forma mais disseminada. A utilização de atorvastatina depende de receituário especial, por se tratar de medicamento incluído no programa de alto custo do governo estadual, e tem sido reservada para casos refratários ou com intolerância à sinvastatina. De modo geral, verificamos a utilização em sua maioria com doses elevadas, seja de sinvastatina ou de atorvastatina.

A grande variabilidade nos níveis lipídicos associados às diversas doses utilizadas indica a necessidade de ajustes nas doses dos medicamentos e, possivelmente, na utilização de estatina com maior potência, reforçando ainda mais a necessidade de utilização de protocolos clínicos bem estabelecidos e de uso institucional para estabelecimento sobre a ação medicamentosa e as doses a serem utilizadas.

Por fim, o número de indivíduos com níveis de LDL-c acima de $190 \mathrm{mg} / \mathrm{dL}$, apesar do uso de estatinas, é significativamente mais elevado do que o reportado na população geral. Esse percentual $(2,4 \%)$ reflete, muito provavelmente, como fator precípuo, a concentração em nível terciário de indivíduos com maior número de comorbidades. Entretanto, considerando ainda o fato de serem mais jovens, isso sugere fortemente a presença de HF, que deve ser investigada de modo sistematizado após se atingirem as doses máximas possíveis de estatina.

\section{Limitações}

Diversas limitações estão presentes neste estudo. Em primeiro lugar, não incluímos todo o perfil lipídico, como os níveis de triglicerídios e colesterol da lipoproteína de alta densidade (HDL-c). Tal ocorrência deveu-se ao fato de ser artigo focado no uso de estatinas, e essas dosagens não interferirem diretamente na sua prescrição. Não foram coletados dados referentes ao uso de ezetimibe, que contribui para a redução dos lipídios mesmo em uso de doses elevadas de estatinas, ${ }^{14}$ pois não faz parte das medicações disponíveis no SUS, apesar de existirem prescrições desse medicamento no receituário ambulatorial, pois alguns pacientes conseguiam adquiri-lo. Outro aspecto limitante foi o fato de não terem 
sido coletadas as comorbidades e dados antropométricos apresentados pelos indivíduos. Infelizmente, em estudos como este, com grande número de dados, a revisão individual dos prontuários não é factível, e o sistema eletrônico de registro de dados (big data) ainda está em fase de implantação, e seu acesso nessa fase preliminar poderia gerar dados errôneos. Desse modo, uma classificação de risco cardiovascular dos pacientes não foi obtida.

\section{Conclusão}

Nesta coorte transversal em um hospital terciário, foi possível verificar que a prescrição de estatinas é disseminada, mas que a obtenção de metas bem estabelecidas de controle de CT e LDL-c não é atingida em grande percentual de indivíduos, e que há, possivelmente, um elevado percentual de portadores de HF que necessitariam ser investigados apropriadamente para melhor definição diagnóstica e de enfoque terapêutico, por suas implicações prognósticas. A adesão institucional a protocolos clínicos diagnósticos e de tratamento mais uniformemente padronizado favoreceria melhor controle de dislipidemias em instituição terciária e a eventual alocação de recursos para prescrição de fármacos novos e efetivos, como os inibidores de PCSK9 em casos selecionados conforme diretrizes clínicas. ${ }^{15}$

\section{Referências}

1. Akioyamen LE, Genest], Shan SD, Reel RL, Albaum JM, Chu A, et al. Estimating the prevalence of heterozygous familial hypercholesterolaemia: a systematic review and meta-analysis. BMJ Open. 2017;7(9):e016461.

2. Ribeiro RA, Duncan BB, Ziegelmann PK, Stella SF, Vieira JLC, Restelatto LMF, et al. Cost-effectiveness of high, moderate and low-dose statins in the prevention of vascular events in the Brazilian public health system. Arq Bras Cardiol. 2015;104(1):32-44

3. Araujo DV, Souza CPR, Bahia LR, Rey HCV, Santos Jr B, Tura BR, etal. Analysis of cost-effectiveness of simvastatin versus atorvastatin in the secondary prevention of cardiovascular events within the Brazilian public healthcare system. Value Health. 2011;14(5 Suppl 1):S29-32.

4. Bonfim MR, Hansen A, Turi BC, Zanini GS, Oliveira ASB, Amaral SL, et al. Adherence to statin treatment and associated factors in female users from the Unified Health System (SUS). Rev Esc Enferm USP. 2014;48(3):477-83.

5. Sabatine MS, Giugliano RP, Keech AC, Honarpour N, Wiviott SD, Murphy SA, etal. Evolocumab and clinical outcomes in patients with cardiovascular disease. N Engl JMed. 2017;376(18):1713-22.

6. Schwartz GG, Steg PG, SzarekM, Bhatt DL, Bittner VA, Diaz R, etal. Alirocumab and cardiovascular outcomes after acute coronary syndrome. N Engl J Med. 2018;379(22):2097-2107.

7. Vannucchi H, dos Santos JE, Marchini JS et al. Lipidograms in patients at the Clinical Hospital of the Ribeirao Preto Medical School. AMB Rev Assoc Med Bras. 1981;27(9):261-3.

8. Pant P, Hamal PK, Gurung D, Upreti K, Subedi K. Lipid profile in a tertiary care center. JNMA J Nepal Med Assoc. 2010;49(178):147-50.

\section{Contribuição dos autores}

Concepção e desenho da pesquisa: Schmidt A, Maciel BC; Obtenção de dados: Moreira HT, Volpe GJ, Foschini VB, Lascala TF; Análise e interpretação dos dados: Schmidt A, Volpe GJ; Análise estatística: Schmidt A, Moreira HT, Volpe G), Foschini VB; Redação do manuscrito: Schmidt A, Maciel BC, Marin-Neto JA; Revisão crítica do manuscrito quanto ao conteúdo intelectual importante: Moreira HT, Volpe GJ, Foschini VB, Romano MMD, Simões MV, Santos JE, Maciel BC, Marin-Neto JA.

\section{Potencial conflito de interesses}

Os autores declaram não haver conflito de interesses pertinentes.

\section{Fontes de financiamento}

O presente estudo não contou com fontes de financiamento externas.

\section{Vinculação acadêmica}

Não há vinculação deste estudo a programas de pósgraduação.

9. Haymana C, Berlik H, Gunes $Y$ et al. Identifying undiagnosed or undertreated patients with familial hypercholesterolemia from the laboratory records of a tertiary medical center. Turk Kardiyol Dern Ars. 2017;45(8):731-8.

10. Faludi AA, Izar MCO, Saraiva JFK, Chacra APM, Bianco HT, Afiune Neto A, et al.,Sociedade Brasileira de Cardiologia. Atualização da Diretriz Brasileira de Dislipidemias e Prevenção da Aterosclerose - 2017. Arq Bras Cardiol. 2017;109(2 Supl 1):1-76.

11. Range JT, LaFontaine PR, Ryder PT, Polston M. Factors associated with adherence to statin medications of patients enrolled in a self-insured university health plan. Clin Ther. 2018;40(10):1692-1700.

12. Carroll MD, Fryar CD, Nguyen DT. Total and high-density lipoprotein cholesterol in adults: United States, 2015-2016. NCHS Data Brief. 2017 Oct; (290):1-8.

13. Gottlieb S, Harpaz D, Shotan A, Boyko V, Leor J, Cohen M, et al. Sex differences in management and outcome after acute myocardial infarction in the 1990s: A prospective observational communitybased study. Israeli Thrombolytic Survey Group. Circulation. 2000;102(20):2484-90.

14. Cannon CP, Blazing MA, Giugliano RP, McCagg A, White JA, Theroux P, et al. Ezetimibe added to statin therapy after acute coronary syndromes. N Eng J Med. 2015;372(25):2387-97.

15. Santos RD, Gagliardi AC, Xavier HT, Casella Filho A, Araújo DB, Cesena FY, et al. First Brazilian Guidelines for Familial Hypercholesterolemia. Arq Bras Cardiol. 2012;99(2 Suppl 2):1-28. 